\title{
A Minimalist Approach towards Protein Recognition by Epitope Transfer from Functionally Evolved Beta-Sheet Surfaces
}

Srivats Rajagopal, Scott C. Meyer, Aaron Goldman, Min Zhou and Indraneel Ghosh*

Contributions from the Department of Chemistry, University of Arizona, Tucson, AZ 85721

Email: ghosh@email.arizona.edu

\section{Supplementary Information}

\section{General Materials and Methods}

All restriction enzymes, Pfu and Taq polymerases, DNA ligase, and dNTPs were obtained from New England Biolabs (NEB).

\section{Library construction}

The details of the library construction are listed in the Materials and Methods section of the manuscript. The primers used to create the library are as follows:

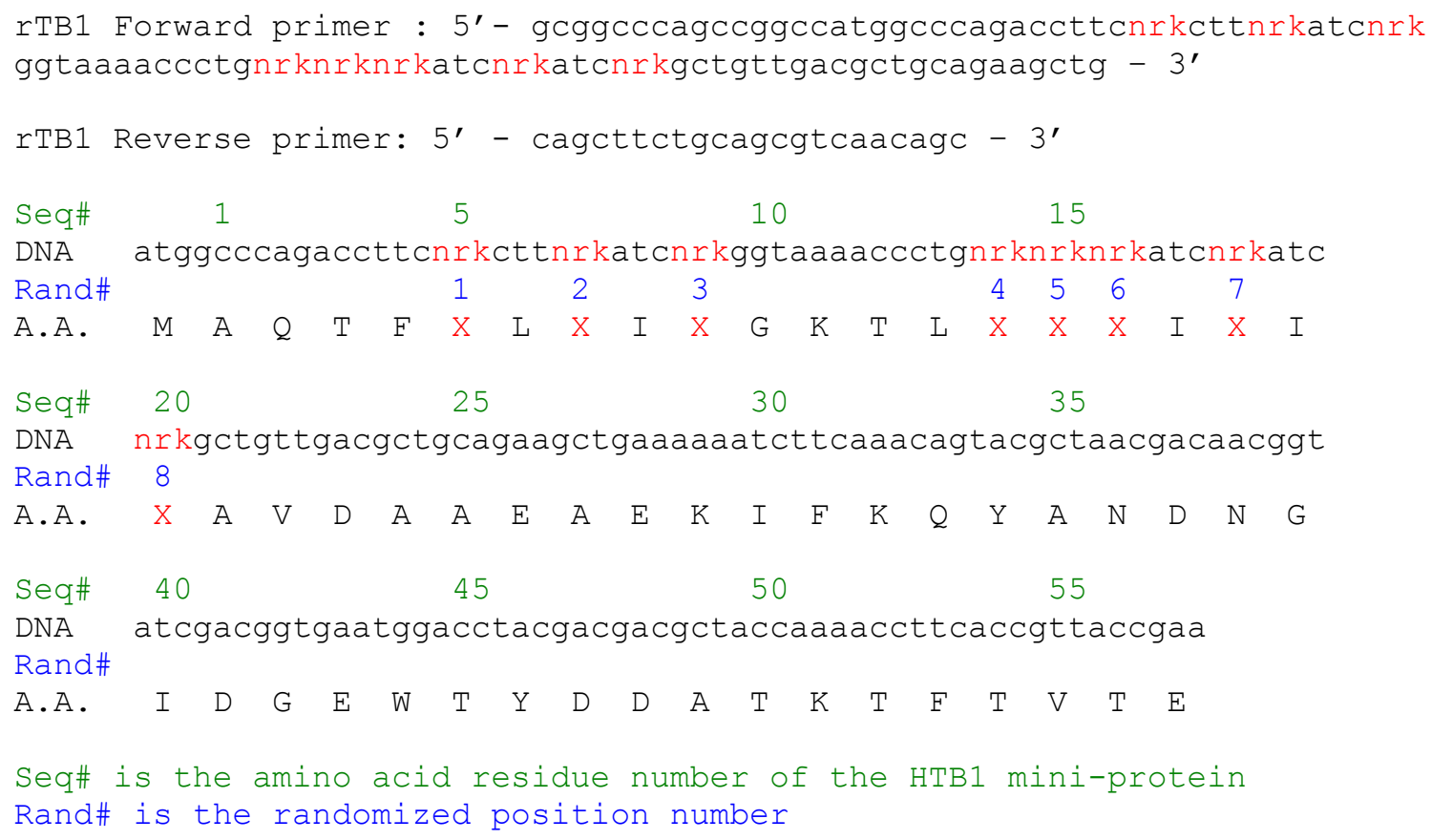

Figure S1. The primers used to create the rHTB1 library cassette. The DNA sequence and the translated amino acid sequence of the HTB1 library are also shown. The 8 mutational sites are depicted in red. 


\section{Primer Sequences}

Protein expression: All selected mutants were sub-cloned into the pQE30 hexa his-tag expression vector (Qiagen) using appropriate primers and confirmed by DNA sequencing. The primers used to sub-clone the B1 mutants are as follows:

Forward primer (9sr4 and 9sr8):

5'-gcatccgcatgcgcagctcactatatggcccagaccttc-3'

Forward primer (all others):

5'-cgcggatccgcagctcattatatgaccttcaagcttatcatcaac- 3'

Reverse primer:

5'-gtatctccccgggctattcggtaacggtgaaggttttg-3'

All mutants except for 9sr4 and 9sr8 were cloned into the BamHI site of pQE-30. Due to an internal BamHI site, these mutants were cloned into the SphI site, which produced a Cys N-terminal to HTB1 sequence. These Cys residues were utilized for labeling in sedimentation equilibrium analysis, but all other assays were carried out with the Ser mutant that was constructed by site-directed mutagenesis. 9sr15C was also constructed via site-directed mutagenesis.

The primers for these mutations are as follows:

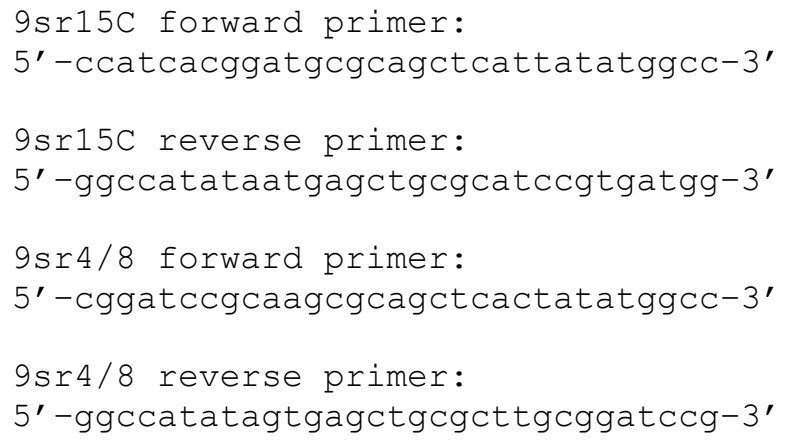

The Y1-Ala and Y5-Ala single alanine mutations were incorporated employing the following primers:

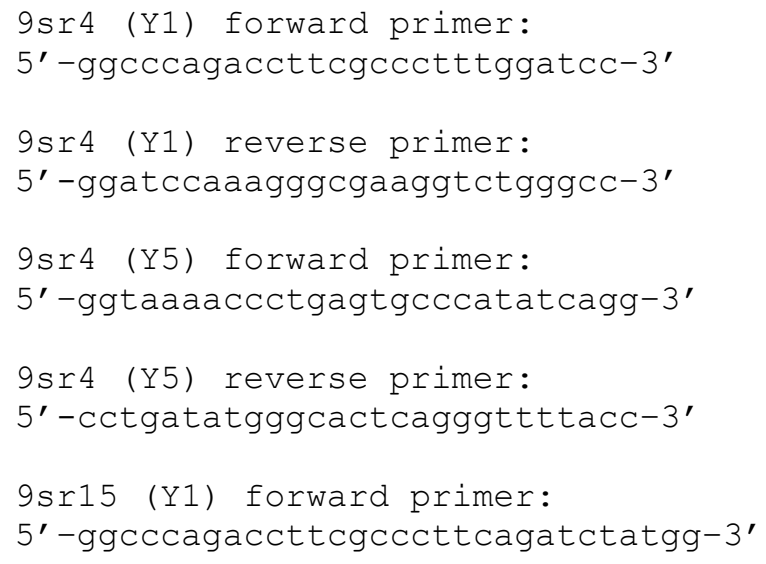


9sr15 (Y1) reverse primer:

5'-ccatagatctgaagggcgaaggtctgggcc-3'

9sr15 (Y5) forward primer:

5'-ggtaaaaccctgggtgcctatatcgagatcc-3'

9sr15 (Y5) reverse primer:

5' -ggatctcgatataggcacccagggttttacc-3'

Figure S2. The primers used in the cloning and site-directed mutagenesis of the selected thrombin-binding HTB1 mutants

Figure S3. Complete amino acid sequences of the HTB1 mutant proteins. The 8 mutational sites located on the first two beta strands are depicted in red. The hexahistidine tag is shown in blue. 


\section{Protein Purification}

All proteins were purified by IMAC with each eluting at $0.5 \mathrm{M}$ imidazole. The imidazole fractions containing our proteins of interest were further purified over a sizeexclusion column (Superdex 75 16/60, Amersham Pharmacia). Typical purified protein yields are $40-60 \mathrm{mg} / \mathrm{L}$.

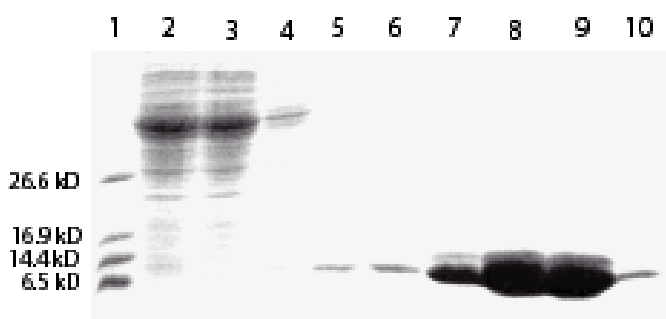

Figure S4. Coomassie stained SDS-PAGE of the Ni-affinity purification profile of 9SR4, an HTB1 variant selected to bind thrombin. Lane 1: Molecular weight markers; Lane 2: flow through; Lanes 3-6: washes; and Lanes 7-10: 0.5 M imidazole elutions.

Following a size-exclusion column purification, the molecular weights of all the proteins was determined by MALDI mass spectrometry.

Table S1. Mass Spectroscopy of HTB1 mutant proteins

\begin{tabular}{|c|c|c|}
\hline Protein & Calculated MW (Da) & Mass Spec Observed MW (Da) \\
\hline HTB1 & 8300.1 & 8300 \\
\hline 9 sr4 & 8723.6 & 8725 \\
\hline 9 sr8 & 8743.4 & 8745 \\
\hline 9 sr15 & 8504.3 & 8505 \\
\hline 9sr19 & 8543.3 & 8549 \\
\hline 9sr4C-Alexa Fluor 488 & 9444.2 & 9424 \\
\hline 9sr15C-Alexa Fluor 488 & 9240.9 & 9126 \\
\hline
\end{tabular}

\section{Sedimentation Equilibrium analysis}

The experimental details of the sedimentation equilibrium analysis are described in the Materials and Methods section of the manuscript.

Labeled 9sr4C protein - (9sr4C-Alexa Fluor 488) with Thrombin.

In the data analysis (rotor speed at $15000 \mathrm{rpm}$ ), the molecular weights of 9sr4C-Alexa Fluor 488 was constrained $(9420 \mathrm{Da})$ along with the molecular weight of the resultant 1:1 complex of 9sr4C-Alexa Fluor 488 with thrombin $(45920 \mathrm{Da})$. A fraction for the bound complex was obtained which allowed an estimate of the equilibrium dissociation constant $\left(\mathrm{K}_{\mathrm{d}}\right)$ for the interaction between 9sr4C-Alexa Fluor 488 and thrombin. On constraining only the molecular weight of 9sr4C-Alexa Fluor 488 (9420 Da), the curve fit and the residuals did not fit the data. A separate control experiment of 9sr4C-Alexa Fluor 488 was simultaneously performed, and a molecular weight of $9375 \mathrm{Da}$ was obtained, which is consistent with the calculated molecular weight of $9420 \mathrm{Da}$. The data was analyzed using SEDEQ 4.1 (Allen Minton, Laboratory of Biochemical Pharmacology, NIH). 
The results of the sedimentation equilibrium data are tabulated below (Table S2) and representative data are shown in Figure S5.

\section{Sedimentation Equilibrium Traces for 9sr4C-Alexa Fluor 488}

1)

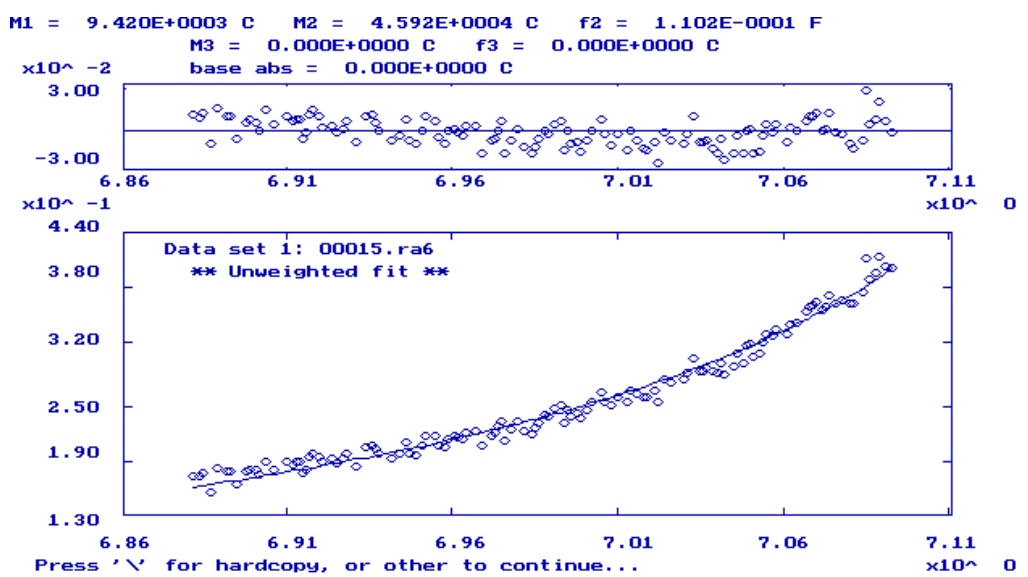

2)

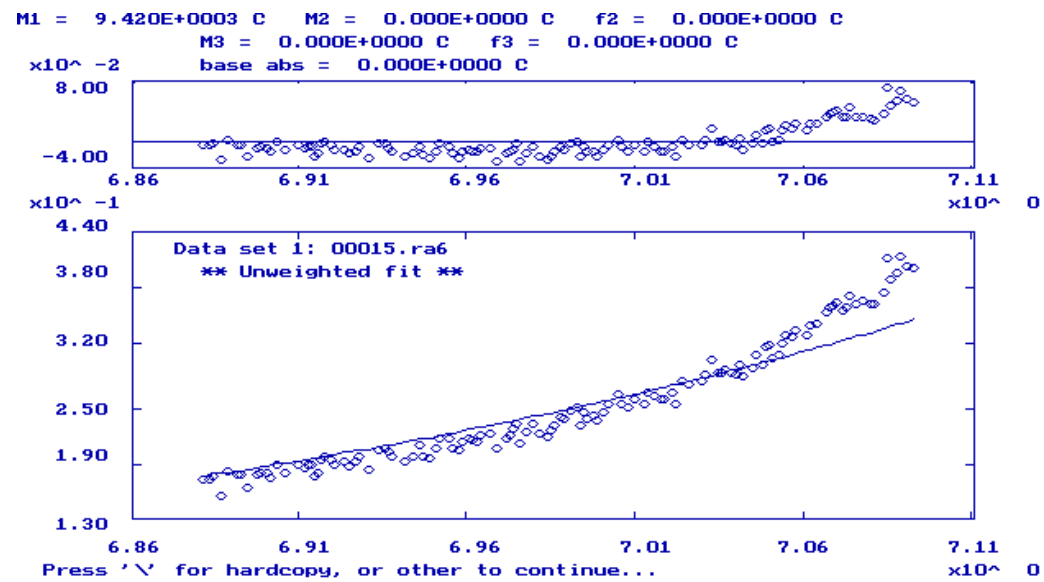

3)

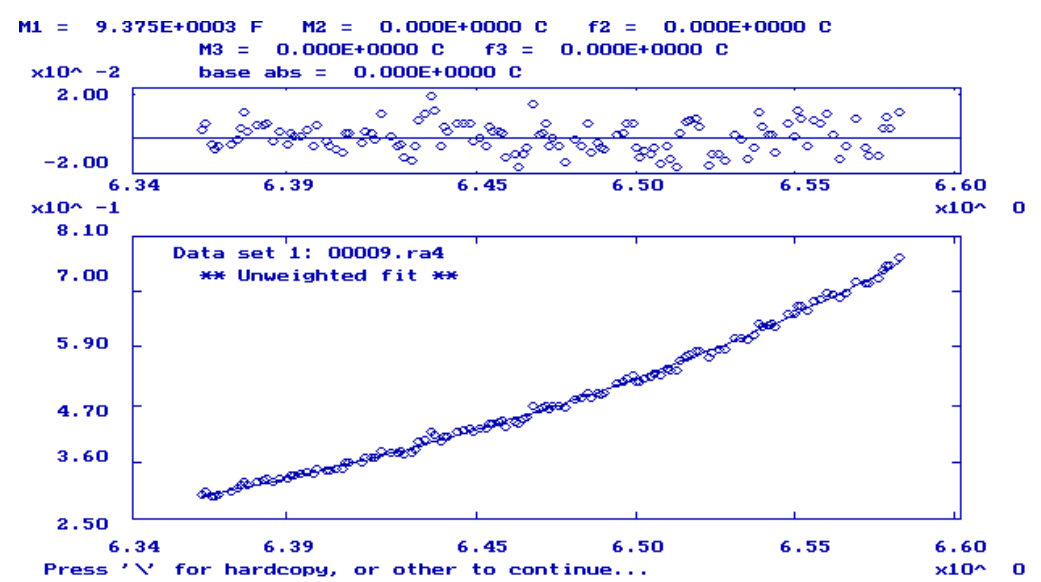

Figure S5. Sedimentation Equilibrium data at pH 8.0 in $20 \mathrm{mM}$ Tris, $20 \mathrm{mM}$ $\mathrm{NaCl}$. Fits are depicted for 1) 9sr4C-Alexa Fluor 488 and 9sr4C-Alexa Fluor 488 with thrombin (resultant labeled complex). 2) 9sr4C-Alexa Fluor 488 force fit to 9420 Da. 3) Control experiment of 9sr4C-Alexa Fluor 488. 
Table S2. Sedimentation Equilibrium Data

\begin{tabular}{|c|c|c|l|}
\hline Curve Fit \# & MW (Da) & Fraction & \multicolumn{1}{c|}{ Conclusion } \\
\hline 1 & $\begin{array}{c}9420 \\
45920\end{array}$ & 0.11 & $\begin{array}{l}\text { Fraction for the labeled bound complex } \\
\text { enables an estimate of the } \mathrm{K}_{\mathrm{d}}(14 \mu \mathrm{M})\end{array}$ \\
\hline 2 & 9420 & & Curve does not fit the data \\
\hline 3 & $\begin{array}{c}9420 \text { calculated } \\
9375 \text { observed }\end{array}$ & & $\begin{array}{l}\text { Confirms the molecular weight of the } \\
\text { labeled mini-protein in the sedimentation } \\
\text { equilibrium context }\end{array}$ \\
\hline
\end{tabular}

The dissociation constant $\left(\mathrm{K}_{\mathrm{d}}\right)$ for 9SR4 and thrombin was calculated to be $13.5 \mu \mathrm{M}$ using the following equation:

$$
K_{d}=\frac{\left([9 S R 4]_{T}-[9 S R 4]_{T} f_{c}\right)\left([T h r]_{T}-[9 S R 4]_{T} f_{c}\right)}{[9 S R 4]_{T} f_{c}}
$$

Where:

$K_{d}=$ Dissociation constant of the thrombin-labeled 9SR4 interaction $[9 S R 4]_{T}=$ Total concentration of labeled 9SR4 $(3.9 \mu \mathrm{M})$

$[T h r]_{T}=$ Total concentration of thrombin $(2.1 \mu \mathrm{M})$

$f_{c}=$ Fraction of labeled 9SR4 that is bound to thrombin, from the best fit of the sedimentation equilibrium data

Similarly, the $\mathrm{K}_{\mathrm{d}}$ for $9 \mathrm{SR} 15$ was determined to be $9.6 \mu \mathrm{M}$, where $[9 S R 15]_{T}$ was $7.8 \mu \mathrm{M}$; $[T h r]_{T}$ was $4.2 \mu \mathrm{M}$; and $f_{c}$ was found to be 0.21 from sedimentation equilibrium experiments.

\section{Specificity of $9 \mathrm{sr} 4$ for thrombin}

We investigated whether 9SR4 was specific for thrombin or if it inhibited other serine proteases as well. The proteolytic assay was revisited with trypsin. 9sr4 weakly inhibited trypsin, which suggests that 9SR4 is selective for its target and not any general protease. The results of the experiment are tabulated below.

Table S3. Comparison of activity of 9SR4 assayed against trypsin and thrombin.

\begin{tabular}{ccc}
$\begin{array}{c}\text { Concentration } \\
(\mu \mathrm{M})\end{array}$ & $\begin{array}{c}\text { Trypsin with 9sr4 } \\
\text { (Normalized Inhibition) }\end{array}$ & $\begin{array}{c}\text { Thrombin with 9sr4 } \\
\text { (Normalized Inhibition) }\end{array}$ \\
\hline 100 & 0.08 & 0.915 \\
\hline
\end{tabular}




\section{Circular Dichroism Spectroscopy}

CD spectroscopy details are listed in the Materials and Methods section of the manuscript. The CD spectra of the 9sr4 and 9sr15 (Y1 and Y5) alanine mutants are depicted below. These alanine mutants are well structured in solution and have spectra very similar to their respective parent proteins $-9 \mathrm{sr} 4$ and $9 \mathrm{sr} 15$.

A)

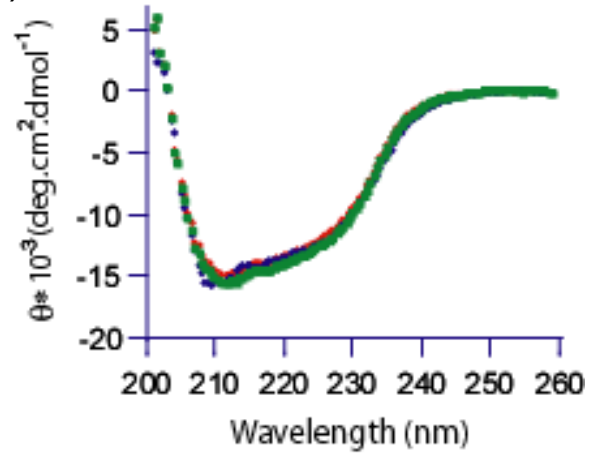

B)

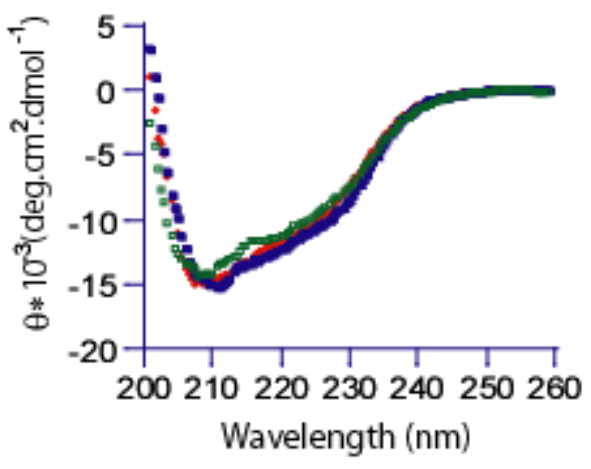

Figure S6. Far UV-circular dichroism of A) protein 9sr4 family and B) 9SR15 family. A) The CD spectra of $9 \mathrm{sr} 4$ (green) is shown with the alanine mutants $-9 \mathrm{sr} 4(\mathrm{Y} 1 \mathrm{~A})$ (in blue) and $9 \mathrm{sr} 4(\mathrm{Y} 5 \mathrm{~A}$ ) (in red). B) $9 \mathrm{sr} 15$ (blue) is shown with the alanine mutants - $9 \operatorname{sr} 15(\mathrm{Y} 1 \mathrm{~A})$ (in green) and $9 \operatorname{sr} 15(\mathrm{Y} 5 \mathrm{~A})$ (in red).

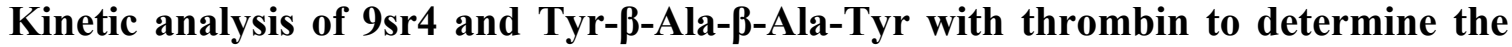 mode of inhibition}

Thrombin was subjected to a kinetic analysis with both the parent protein $9 \mathrm{sr} 4$ and the tetrapeptide - Tyr- $\beta$-Ala- $\beta$-Ala-Tyr. $9 \mathrm{sr} 4$ was used at three different concentrations $-5 \mu \mathrm{M}, 25 \mu \mathrm{M}$, and $50 \mu \mathrm{M}$. The resultant kinetic data sets were fitted globally to three different models: competitive inhibition, uncompetitive inhibition, and non-competitive inhibition using equations that describe these models (SigmaPlot).

The data suggests a non-competitive mode of inhibition for 9sr4 and the tetrapeptide, Tyr- $\beta$-Ala- $\beta$-Ala-Tyr against thrombin. The AICc value is the least for the global fit to equation $\mathbf{S 3}$, indicating that the non-competitive model is the best fit for both cases.

$$
v=\frac{V_{\max }}{1+\left(\frac{K_{m}}{[S]}\right)\left(1+\frac{I}{K_{i}}\right)}
$$

Eq. S1: Competitive Inhibition

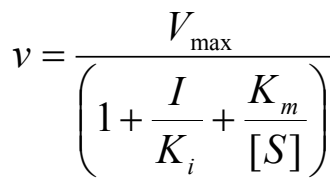

Eq. S2: Uncompetitive Inhibition
$v=\frac{V_{\max }}{\left(1+\frac{I}{K_{i}}\right)\left(1+\frac{K_{m}}{[S]}\right)}$

Eq. S3: Non-competitive Inhibition 


\section{Determination of Mode of Inhibition for 9sr4}

\section{A) Competitive Inhibition}

Parameters

$\mathrm{V}_{\max } \quad 6510.3 \pm 365$

$\mathrm{K}_{\mathrm{m}} \quad 13.42 \pm 4.2$

$\mathrm{K}_{\mathrm{i}} \quad 3.61 \pm 1.04$

Goodness of Fit

Degrees of Freedom 29

AICc $\quad 405.8$

$\mathrm{R}^{2} \quad 0.984$

Sum of Squares $\quad 7.67 \mathrm{e}+6$

Sy.x $\quad 514.4$

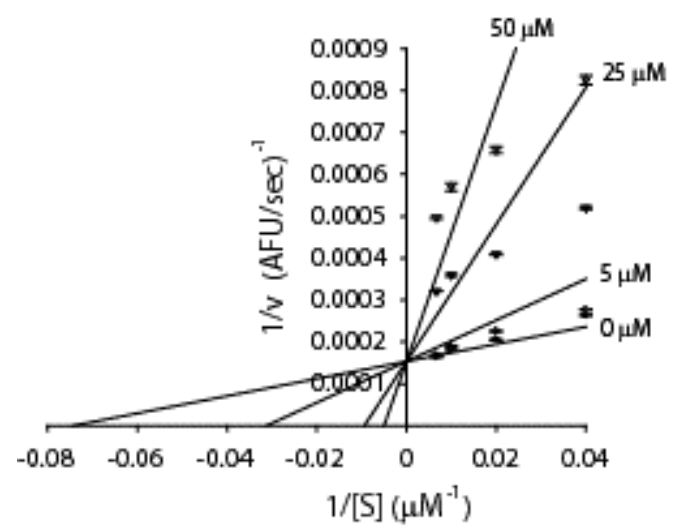

\section{B) Uncompetitive Inhibition}

Parameters

$\mathrm{V}_{\max } \quad 7415.5 \pm 295$

$\mathrm{K}_{\mathrm{m}} \quad 24.5 \pm 3.4$

$\mathrm{K}_{\mathrm{i}} \quad 18.9 \pm 1.5$

Goodness of Fit

Degrees of Freedom 29

AICc $\quad 376.5$

$\mathrm{R}^{2} \quad 0.994$

Sum of Squares $\quad 3.06 \mathrm{e}+6$

Sy.x 324.9

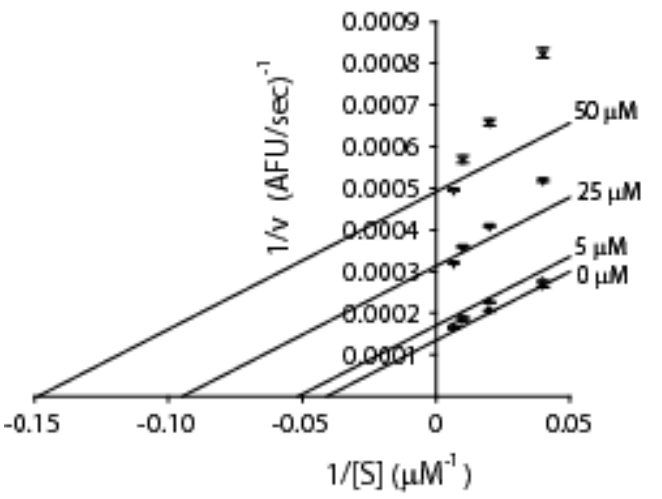

C) Non-competitive Inhibition

Parameters

$\mathrm{V}_{\max } \quad 7164.6 \pm 216$

$\mathrm{K}_{\mathrm{m}} \quad 20.9 \pm 2.4$

$\mathrm{K}_{\mathrm{i}} \quad 25.2 \pm 1.6$

Goodness of Fit

Degrees of Freedom 29

AICc $\quad 367.5$

$\mathrm{R}^{2}$

0.995

Sum of Squares

$2.31 \mathrm{e}+6$

Sy.x

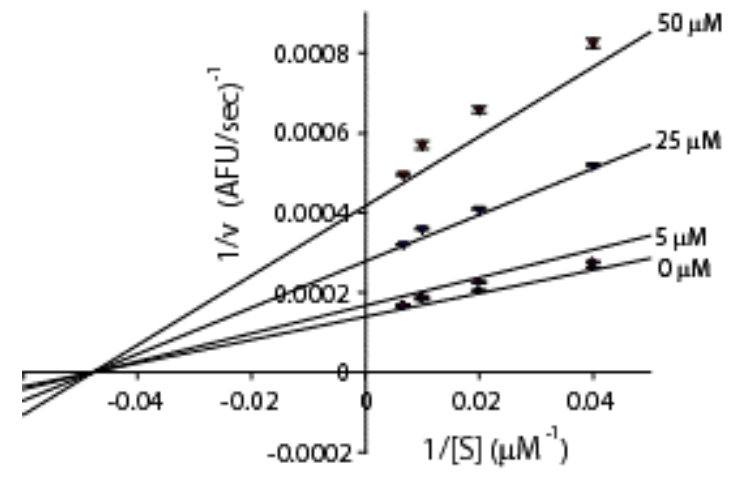

Figure S7. Thrombin was subjected to a kinetic analysis with the HTB1 mutant 9sr4. The protein was used at three different concentrations $-5 \mu \mathrm{M}, 25 \mu \mathrm{M}$, and 50 $\mu \mathrm{M}$ and subjected to analysis using different kinetic models - competitive, uncompetitive, and non-competitive inhibition. 


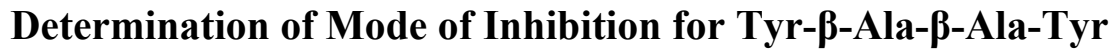

\section{A) Competitive Inhibition}

Parameters

$\mathrm{V}_{\text {max }} \quad 6307 \pm 335$

$\mathrm{K}_{\mathrm{m}} \quad 14.64 \pm 4.5$

$\mathrm{K}_{\mathrm{i}} \quad 90.1 \pm 24.5$

Goodness of Fit

Degrees of Freedom 37

AICc $\quad 505.7$

$\mathrm{R}^{2} \quad 0.981$

Sum of Squares $\quad 9.87 \mathrm{e}+6$

Sy.x $\quad 516.4$

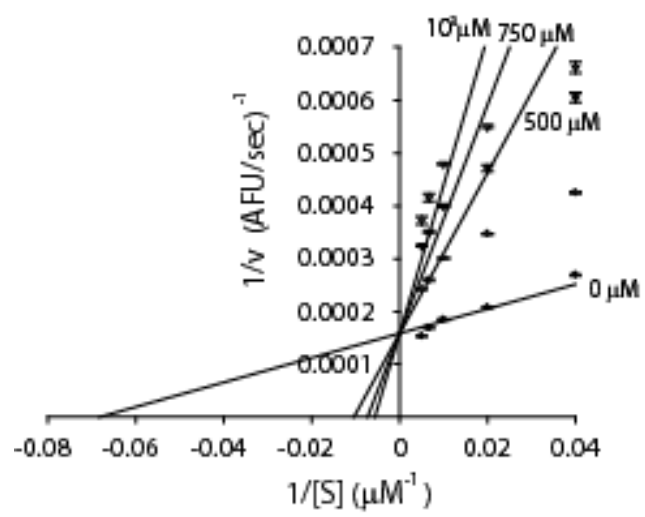

\section{B) Uncompetitive Inhibition}

Parameters

$\mathrm{V}_{\max } \quad 7412.5 \pm 243$

$\mathrm{K}_{\mathrm{m}} \quad 30.46 \pm 3.6$

$\mathrm{K}_{\mathrm{i}} \quad 538.7 \pm 31.5$

Goodness of Fit

Degrees of Freedom

AICc

37

$\mathrm{R}^{2}$

457.9

Sum of Squares

0.994

Sy.x

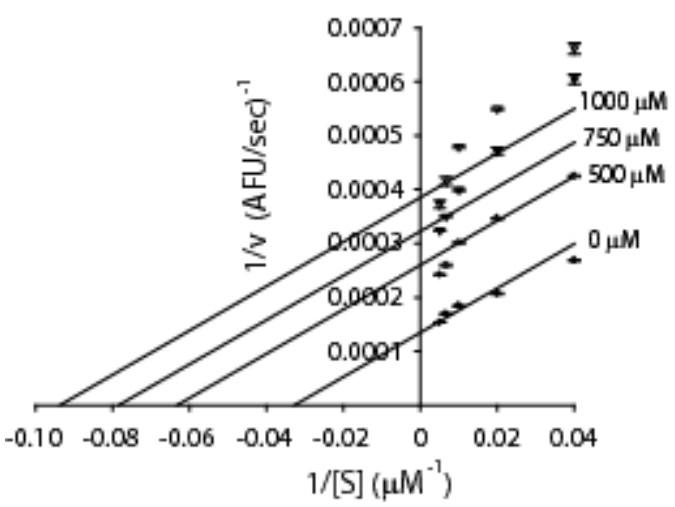

\section{C) Non-competitive Inhibition}

Parameters

$\mathrm{V}_{\max } \quad 7098.5 \pm 141$

$\mathrm{K}_{\mathrm{m}} \quad 24.3 \pm 1.9$

$\mathrm{K}_{\mathrm{i}} \quad 704.5 \pm 26$

Goodness of Fit

Degrees of Freedom

AICc

$\mathrm{R}^{2}$

Sum of Squares

Sy.x
37

431.4

0.997

$1.54 \mathrm{e}+6$

203.8

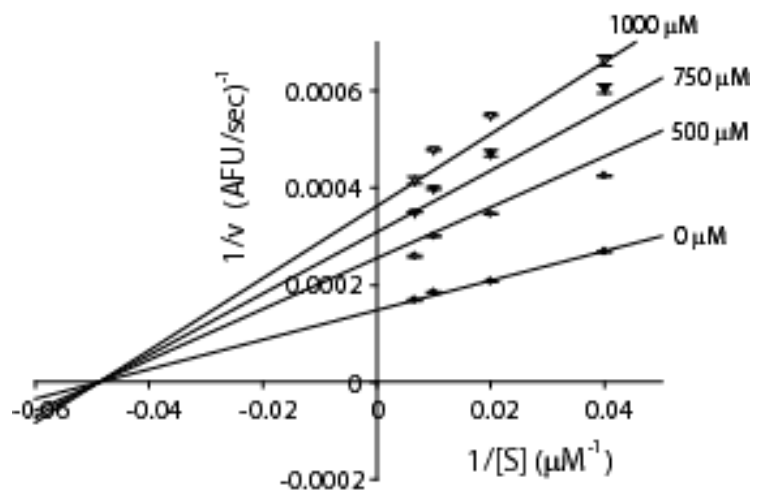

Figure S8. Thrombin was subjected to a kinetic analysis with the tetrapeptide - Tyr$\beta$-Ala- $\beta$-Ala-Tyr. The tetrapeptide was used at three different concentrations -500 $\mu \mathrm{M}, 750 \mu \mathrm{M}$, and $1000 \mu \mathrm{M}$ and subjected to analysis using different kinetic models competitive, uncompetitive, and non-competitive inhibition. 


\section{ANS-binding studies}

The propensity of a peptide to aggregate can be probed by change in fluorescence of the dye, 8-anilino-1-naphthalene sulfonic acid (ANS). Typically, the fluorescence of ANS increases upon interaction with an apolar environment and has been used to measure the critical micellar concentration (CMC) of detergents such as sodium dodecyl sulfate (SDS) $)^{1,2}$ and in measuring hydrophobic environments in proteins and aggregating peptides. $^{3,4}$ A stock solution of ANS (Sigma A-1028) (1.55 mM) was prepared using sterile filtered $(0.2 \mu \mathrm{m})$ nanopure water and was used immediately. The final concentration of ANS in all experiments was $10 \mu \mathrm{M}$ and sodium dodecyl sulfate (SDS) was used as a control. All experiments were performed in $20 \mathrm{mM}$ tris containing $20 \mathrm{mM}$ $\mathrm{NaCl}$ at $\mathrm{pH} 8.0$ at room temperature. Fluorescence measurements were performed using a Photon Technology International (PTI) spectrofluorimeter. The excitation wavelength was $370 \mathrm{~nm}$ and the emission was recorded from 420 to $600 \mathrm{~nm}$. The excitation and emission slit widths were $10 \mathrm{~nm}$ and $12 \mathrm{~nm}$, respectively. Fluorescence spectra of $10 \mu \mathrm{M}$ ANS in the presence of increasing amounts of the tetrapeptide Tyr- $\beta$-Ala- $\beta$-Ala-Tyr (250 $\mu \mathrm{M}-1250 \mu \mathrm{M})$ and SDS $(0.1 \mathrm{mM}-15 \mathrm{mM})$ were separately determined after incubating the samples with dye for 2 hours at room temperature. Each spectrum was recorded twice and averaged. The emission wavelength maximum for ANS with the tetrapeptide was $470 \mathrm{~nm}$ and for ANS with SDS was $485 \mathrm{~nm}$. A plot of AFU at the respective emission wavelength maxima versus increasing concentrations for both the tetrapeptide and SDS with ANS were plotted separately (Fig. S10A and S10B), and the fluorescence emission spectra between $420 \mathrm{~nm}$ to $600 \mathrm{~nm}$ for the tetrapeptide (Tyr- $\beta$-Ala- $\beta$-Ala-Tyr) and SDS are shown as insets. There is no evidence of tetrapeptide aggregation at concentrations utilized in the thrombin inhibition data, since there is no increase in fluorescence upon binding with the ANS dye. The control, SDS, causes a large increase in ANS fluorescence intensity with increasing concentrations in agreement with literature data. ${ }^{1,2}$
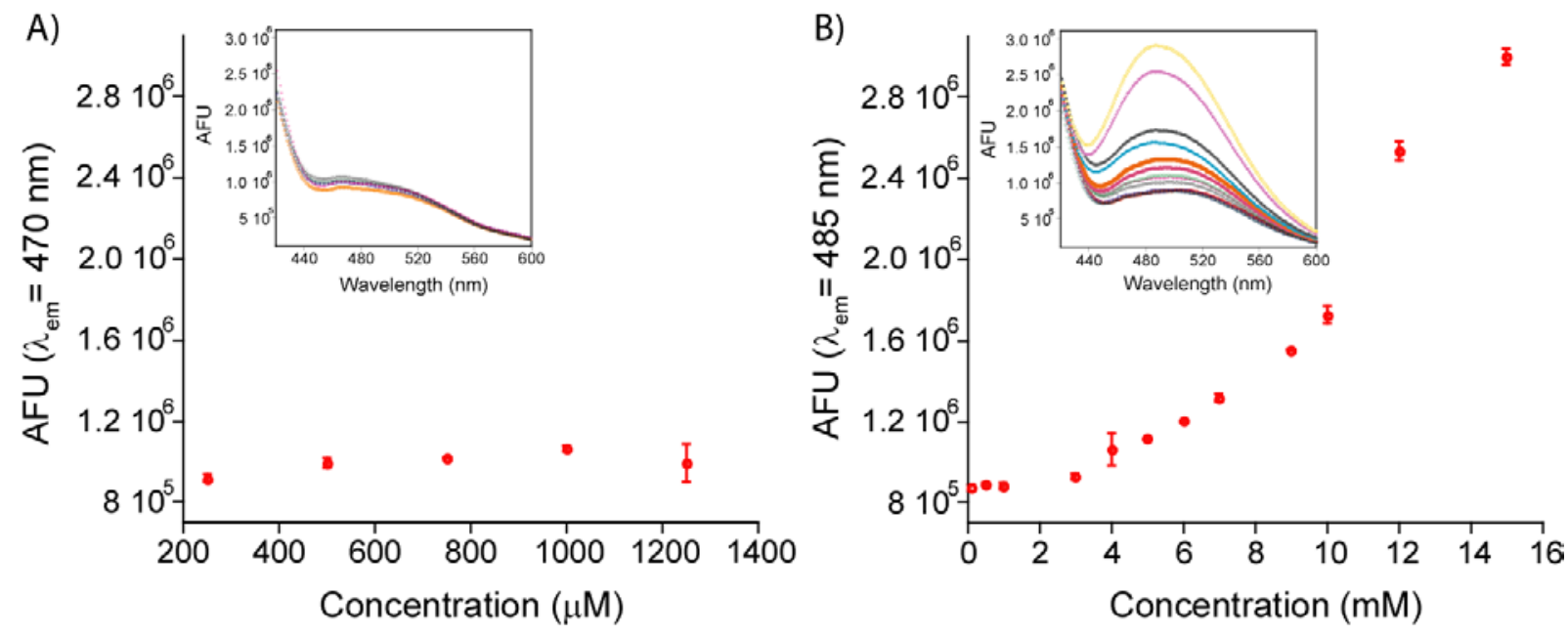

Figure S9. 8-Anilino-1-naphthalene sulfonic acid (ANS) binding. The fluorescence emission of ANS at its maxima with increasing concentrations of A) Tyr- $\beta$-Ala- $\beta$-AlaTyr at $470 \mathrm{~nm}$, and B) SDS at $485 \mathrm{~nm}$. The raw spectra in each case are shown as insets. 


\section{Dynamic Light Scattering (DLS)}

Dynamic light scattering experiments were carried out on the tetrapeptide - Tyr$\beta$-Ala- $\beta$-Ala-Tyr to further test for potential aggregation of peptide. A solution of the tetrapeptide $(1 \mathrm{mM})$ was prepared in the thrombin assay buffer, along with SDS controls. The SDS concentrations were chosen as being below and above the apparent critical micellar concentration (CMC) of SDS as indicated by the ANS dye assay from above. Thus, we chose to test $1 \mathrm{mM}$ and $10 \mathrm{mM}$ SDS. Light scattering data was collected at a $90^{\circ}$ angle on a BI8000 autocorrelator from Brookhaven Instrument Corp. ${ }^{5}$ Data was fit using a non-negatively constrained least squares method, with a range of 3 to $500 \mathrm{~nm}$. The DLA data for $1 \mathrm{mM}$ peptide was identical to that of the buffer background whereas the $10 \mathrm{mM}$ SDS control clearly showed aggregation as also seen in the ANS binding experiments.

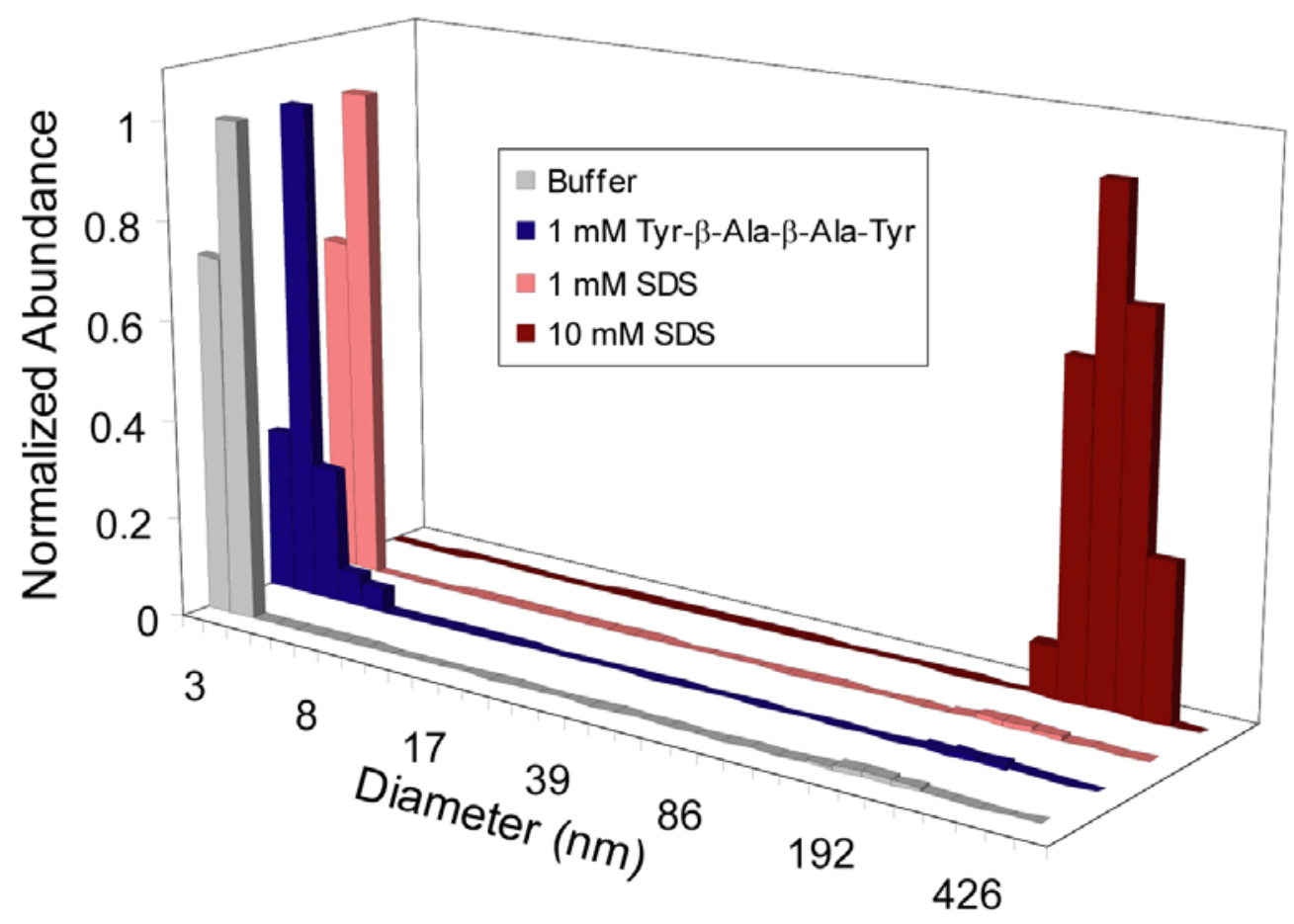

Figure S10. Dynamic Light Scattering (DLS). DLS readings of the tetrapeptide at 1 $\mathrm{mM}$ show particle abundance profiles similar to those of the buffer background, indicating that no significant aggregation is taking place under assay conditions. Also shown are abundance profiles for SDS at $1 \mathrm{mM}$ (below the apparent CMC) and 10 $\mathrm{mM}$ (above the apparent $\mathrm{CMC}$ ).

\section{Reversibility of Inhibition}

Many non-competitive inhibitors of enzymes display irreversible inhibition ${ }^{6}$ such that enzymatic activity is permanently lost either through covalent modification or by irreversible enzyme denaturation. To determine if our tetrapeptide inhibitor was a reversible or irreversible inhibitor, an exhaustive dialysis experiment was performed. 
Thrombin was incubated in the presence or absence of the tetrapeptide (Tyr- $\beta$-Ala- $\beta$-AlaTyr) at a concentration of $1 \mathrm{mM}$ The enzymatic was measured prior to dialysis (Figure S11). This mixture was dialyzed for $3 \times 8$ hours at $4^{\circ} \mathrm{C}$ in a dialysis cassette $(7 \mathrm{kDa}$ molecular weight cut off, Pierce) in $150 \mathrm{~mL}$ of $20 \mathrm{mM}$ tris, $20 \mathrm{mM} \mathrm{NaCl}, 0.05 \%$ Triton $\mathrm{X}-100$ at $\mathrm{pH}$ 8.0. A control consisting of thrombin $(1 \mathrm{nM})$ and buffer was also dialyzed in the same manner. After dialysis, the thrombin activity was assayed by the addition of the fluorogenic substrate, Benzoyl-Phe-Val-Arg.AMC, (Calbiochem). We observed that the enzymatic activity of thrombin was completely restored when the inhibitor was removed by exhaustive dialysis, demonstrating reversible inhibition.

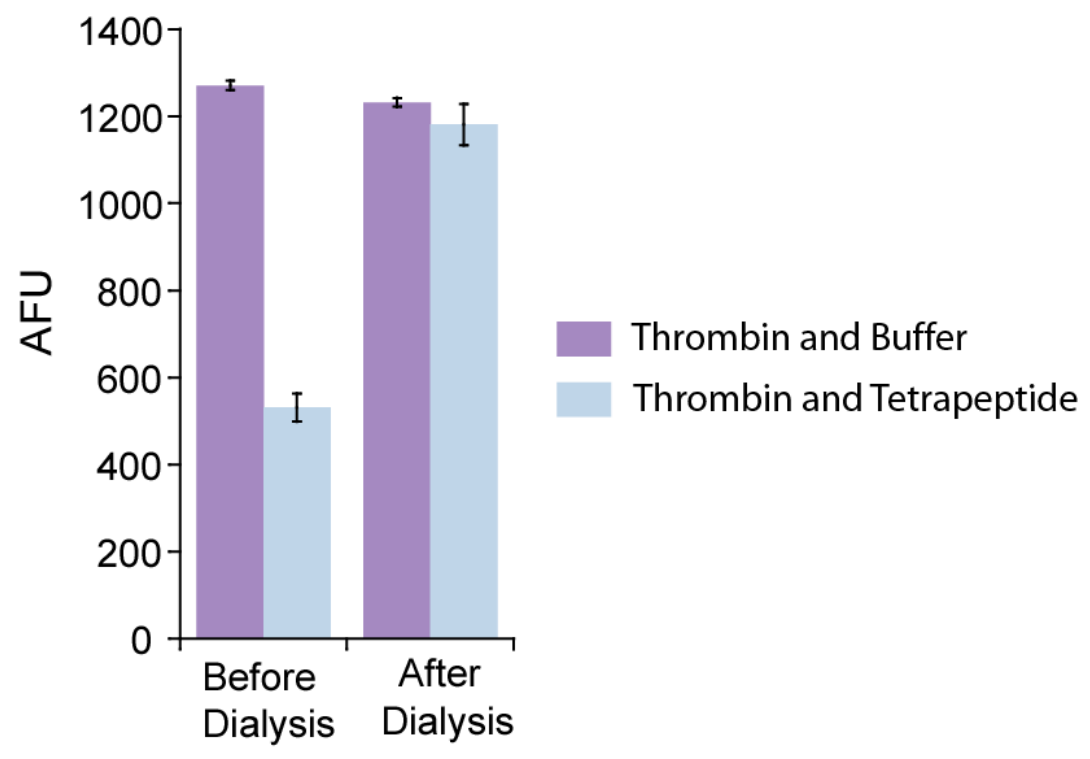

Figure S11. Reversibility of inhibitor binding. Thrombin catalytic activity before and after exhaustive dialysis in the absence or presence of the tetrapeptide, Tyr- $\beta$-Ala- $\beta$-Ala$\operatorname{Tyr}(1 \mathrm{mM})$, demonstrating that binding and inhibition is reversible.

\section{Limited Proteolysis}

A combination of limited proteolysis and mass spectrometry was utilized to generate a binding map of the selected thrombin-binding mutant to thrombin. By comparing the mass spectra for trypsin digested thrombin and 9sr4 separately with the trypsin digests of the corresponding complexes, difference tryptic digest maps of thrombin were generated. Positions on thrombin that were cleaved when free in solution but were not cleaved upon complexation of $9 \mathrm{sr} 4$ were considered to be protected. The positions were then mapped onto a crystal structure of thrombin (Figure S9). For the interaction between thrombin and 9sr4, the thrombin amino acid residues 50, 93, 202, 206 , and 233 were protected. 


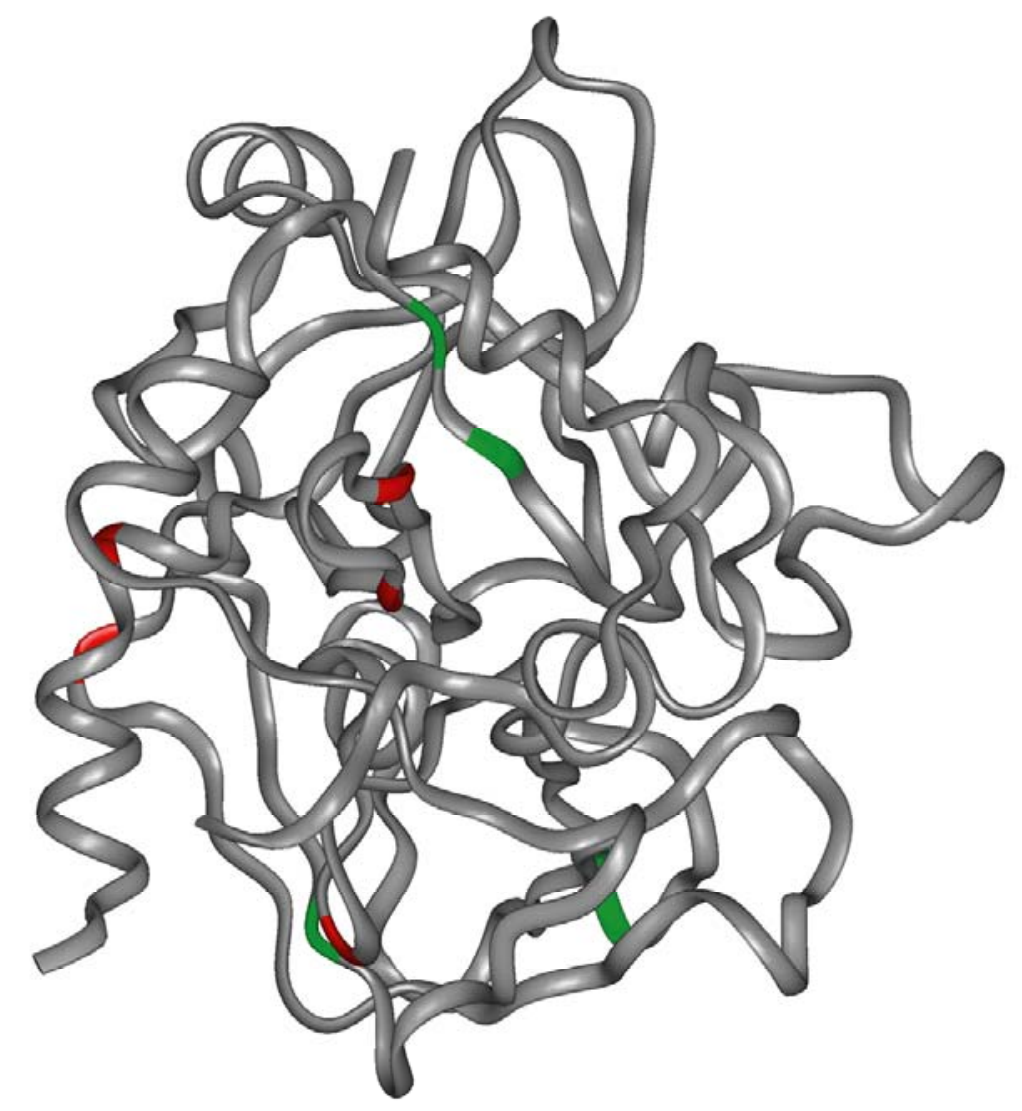

Figure S12. Difference tryptic digest map of thrombin. The structure depicts the protection sites on the surface of thrombin by the HTB1 mutant 9sr4. The protection sites are depicted in red while the unprotected sites (increased cleavage in the presence of inhibitor) are shown in green. $9 \mathrm{sr} 4$ thus appears to localize on the heparin-binding site of thrombin.

\section{References}

(1) Esposito, C. C., P.; Facchiano, A.; Ragone, R. J Colloid Interface Sci 1998, 200, 310-312.

(2) Fisher, L. E.; Engelman, D. M.; Sturgis, J. N. Biophys J 2003, 85, 3097-3105.

(3) Betz, S. F.; Raleigh, D. P.; DeGrado, W. F.; Lovejoy, B.; Anderson, D.; Ogihara, N.; Eisenberg, D. Fold Des 1996, 1, 57-64.

(4) Kayed, R.; Bernhagen, J.; Greenfield, N.; Sweimeh, K.; Brunner, H.; Voelter, W.; Kapurniotu, A. J Mol Biol 1999, 287, 781-796.

(5) Kolchens, S.; Ramaswami, V.; Birgenheier, J.; Nett, L.; O'Brien, D. F. Chem Phys Lipids 1993, 65, 1-10.

(6) Zhu, K.; Cordeiro, M. L.; Atienza, J.; Robinson, W. E., Jr.; Chow, S. A. J Virol 1999, 73, 3309-3316. 


\section{Complete reference $\mathbf{4 2}$ from main manuscript.}

42. Ohren, J. F.; Chen, H.; Pavlovsky, A.; Whitehead, C.; Zhang, E.; Kuffa, P.; Yan, C.; McConnell, P.; Spessard, C.; Banotai, C.; Mueller, W. T.; Delaney, A.; Omer, C.; Sebolt-Leopold, J.; Dudley, D. T.; Leung, I. K.; Flamme, C.; Warmus, J.; Kaufman, M.; Barrett, S.; Tecle, H.; Hasemann, C. A. Nat Struct Mol Biol 2004, 11, 11921197. 